\title{
Pesquisa-ação como método para construção de instrumento de Consulta de Enfermagem à criança
}

\author{
Elisangela Argenta Zanatta ${ }^{1}$, Ingrid Pujol Hanzen ${ }^{2}$, Cheila Karei Siega ${ }^{3}$ \\ Dara Montag Portaluppi ${ }^{4}$ e Denise Antunes de Azambuja Zocche ${ }^{1}$ \\ 1 Departamento de Enfermagem, Mestrado Profissional em Enfermagem na Atenção \\ Primária à Saúde Universidade do Estado de Santa Catarina, Brasil \\ elisangela.zanatta@udesc.br; denise.zocche@udesc.br | https://orcid.org/ 0000-0002-7426- \\ 6472; https://orcid.org/0000-0003-4754-8439 \\ ${ }^{2}$ Secretaria Municipal de Saúde de Chapecó, Santa Catarina, Brasil | \\ ingridhanzen@yahoo.com.br | https://orcid.org/0000-0001-9808-2005 \\ ${ }^{3}$ Serviço Nacional de Aprendizagem Comercial - SENAC, Caçador, Santa Catarina, Brasil | \\ cheilasiega@gmail.com | https://orcid.org/0000-0001-9272-2526 \\ ${ }^{4}$ Programa de Residência Multiprofissional em Saúde da Escola de Saúde Pública do RS, \\ Brasil | dara.portaluppi@hotmail.com | https://orcid.org/0000-0003-4156-9482
}

Resumo: $O$ enfermeiro tem papel relevante no acompanhamento criterioso e sistemático à saúde da criança. A execução periódica desse acompanhamento, por meio da Consulta de Enfermagem, possibilita detectar precocemente agravos e instituir ações de promoção à saúde no âmbito da Atenção Primária à Saúde. Objetivos: construir um instrumento para guiar a Consulta de Enfermagem à criança na Atenção Primária à Saúde, com base na Classificação Internacional para as Práticas de Enfermagem e nas Necessidade Humanas Básicas. Métodos: estudo qualitativo do tipo Pesquisa-ação realizada a partir de cinco etapas, adaptadas do método proposto por Thiollent: fase exploratória, diagnóstico de situação, seminários integradores, planejamento de qualificação dos profissionais enfermeiros, publicização dos resultados. Os dados coletados, por meio de entrevistas e nos seminários integradores, foram organizados seguindo a análise de conteúdo e discutidos com a literatura científica sobre Consulta de Enfermagem. Resultados: os dados revelam que os enfermeiros compreendem a importância da Consulta de Enfermagem à criança contudo, possuem dificuldade para executá-la em todas as suas etapas. Como produto desse estudo, foi construído, de forma coletiva, um instrumento para guiar a Consulta de Enfermagem à criança formado por Histórico de Enfermagem, Diagnósticos de Enfermagem, Intervenções de Enfermagem e Resultados esperados. Conclusões: A pesquisa-ação contribuiu significativamente para subsidiar a reflexão acerca da importância da realização da Consulta de Enfermagem de forma sistematizada e para a construção coletiva de um instrumento para guia-la, auxiliando no processo de trabalho do enfermeiro. Acredita-se que esse instrumento qualifica e otimiza o atendimento à criança, tornando-o mais resolutivo ao mesmo tempo em que auxilia o enfermeiro a tomar decisões seguras, assertivas considerando a criança na sua integralidade.

Palavras-chave: Processo de Enfermagem; Enfermeiros; Atenção Primária à Saúde; Pesquisa Aplicada.

Action Research as Method for Build an Instrument for the Nursing Consultation to Children

Abstract: The nurse has a relevant role in child's health systematic monitoring. The periodical execution of this monitoring, via nursing consultation, allows for the early detection of possible complications and therefore also makes it possible to act upon such findings in primary care. Objectives: build an instrument to guide the Nursing Consultation to children in Primary Health Care based on the International Classification for Nursing Practices and Basic Human Needs. Methods: Action research carried out from five stages, adapted from the method proposed by Thiollent: exploratory phase, situation diagnosis, integrating seminars, planning for the qualification of professional nurses, publication the results. The data collected, through interviews, and in the integrating seminars were organized according to the content analysis and discussed with the scientific literature on nursing consultation. Results: The data reveal that the nurses comprehend the importance of nursing consultations regarding children, however they have difficulties performing it in full. As a product of this study, an instrument for guidance as to how the nursing consultation has to be performed was collectively developed, consisting of Nursing History, Nursing Diagnoses, Nursing Interventions and Expected Results. Conclusions: The action research significantly contributed as a subsidizing factor for the reflections regarding the importance of performing a nursing consultation in a systematic fashion and also for the creation of a guidance instrument towards its application. It is believed that such an instrument qualifies and optimizes childcare, making it more efficient and at the same time helping nurses to take safer, more assertive decisions.

Keywords: Nursing Process; Nurses; Primary Health Care; Applied Research. 


\section{Introdução}

O Ministério da Saúde (MS) do Brasil declara que a atenção à saúde da criança deve ter prioridade de atendimento, deve ser realizada com vistas a um cuidado integral e resolutivo. Os profissionais que atuam na Atenção Primária à Saúde (APS) devem estar preparados e organizados para atender esse público, considerando a importância do acompanhamento sistemático até o segundo ano de vida da criança, com avalições mensais no primeiro e duas no segundo ano (MS, 2012).

No atendimento à criança, o enfermeiro possui como ferramenta de trabalho o Processo de Enfermagem (PE), instituído no Brasil pela resolução 358/2009 do Conselho Federal de Enfermagem (Cofen) que dispõe sobre sua implementação em ambientes públicos ou privados em que ocorre o cuidado de enfermagem (Cofen, 2009). O PE no âmbito da APS corresponde à Consulta de Enfermagem (CE), respaldada pela Lei do Exercício Profissional ( $n^{-}$7498/86) e assegurada pela Resolução do Cofen no 358/2009 (Cofen, 2009).

A CE à criança, objetiva identificar as necessidades de saúde, por meio de um olhar atento e criterioso voltado, especialmente, para o processo de crescimento e desenvolvimento infantil (Goes et.al, 2018; Yakuma et al.,2018).

O enfermeiro é um dos profissionais da APS que mais tem contato com a criança e sua família, a CE oportuniza identificar fragilidades, promover assistência integral e humanizada e, assim contribuir para a redução das morbimortalidades (Moreira \& Gaiva, 2017).

Para contemplar as cinco etapas do PE (Histórico de Enfermagem, Diagnósticos de Enfermagem, Planejamento e Avaliação de Enfermagem), tem-se a Classificação Internacional para a Prática de Enfermagem (CIPE®), um Sistema de Linguagem Padronizada (SLP), que organiza diagnósticos, resultados e intervenções e, ao mesmo tempo, favorece a adoção de um vocabulário padronizado (Garcia, 2017).

Estudos indicam dificuldades para realização da CE à criança, como falta de estrutura física, insumos, equipamentos, sobrecarga de trabalho, pouca oferta de qualificação no âmbito dos serviços de saúde aos enfermeiros e, principalmente, carência de roteiros para guiá-la (Siega, et al., 2020; Ribeiro et al., 2014).

Justifica-se a importância desse estudo por contribuir na construção de um instrumento para guiar a CE à criança, de forma sistematizada, no âmbito da APS. O presente estudo objetivou construir um instrumento para guiar a Consulta de Enfermagem à criança na Atenção Primária à Saúde, com base na Classificação Internacional para as Práticas de Enfermagem e nas Necessidade Humanas Básicas.

\section{Metodologia}

Estudo de abordagem qualitativa, do tipo pesquisa-ação realizado em dois municípios do Estado de Santa Catarina, Brasil. Participaram 26 enfermeiros (15 de um município e 11 do outro) que atuavam no cuidado à criança na APS. Foram excluídos enfermeiros em afastamento por licença ou férias e com menos de seis meses de atuação na APS.

A pesquisa-ação foi conduzida em cinco etapas adaptadas de Thiollent (2011), fase exploratória, diagnóstico de situação, seminários integradores, qualificação dos enfermeiros e publicização.

$\mathrm{Na}$ fase exploratória foi realizada uma Revisão Integrativa (RI) com o objetivo de identificar tecnologias para o acompanhamento da saúde da criança de zero a cinco anos de idade na APS. A RI foi conduzida seguindo um Protocolo: identificação da questão de pesquisa, estruturada a partir do acrômio PICOT; definição dos critérios para inclusão e exclusão dos estudos; seleção e extração dos dados; análise e interpretação dos dados; apresentação dos resultados; discussão dos resultados (Zocche et al., 2020). 
A busca foi realizada no catálogo de Teses e Dissertações da Coordenação de Aperfeiçoamento de Pessoal de Nível Superior (CAPES) on-line. Os Descritores em Ciências da Saúde (DeCS) utilizados foram: "Criança"; "Teoria de Enfermagem"; "Cuidado de enfermagem", "Atenção Primária à Saúde", "Enfermagem", "Tecnologias em Saúde", "Processo de Enfermagem" e o sinônimo "Consulta de Enfermagem". Esses foram cruzados entre si utilizando-se operador booleano $A N D$.

$\mathrm{Na}$ etapa de Diagnóstico de situação foi traçado o perfil epidemiológico da saúde infantil, dos municípios em estudo, por meio dos dados, de domínio público, coletados nos sistemas de informação em saúde: Sistema de Informação de Mortalidade, Sistema de Informação sobre Nascidos Vivos, Sistema de Informações Hospitalares do Sistema Único de Saúde e no prontuário eletrônico de cada município.

Nessa etapa, também, foram entrevistados 26 enfermeiros que atuavam no cuidado à criança na APS no dois municípios, que cumpriram com os critérios de inclusão, visando compreender os significados atribuídos à $\mathrm{CE}$ à criança, levantar subsídios para a elaboração do instrumento de CE.

Na sequência foram realizados quatro Seminários integradores em cada município, com intervalo de 15 dias entre um e outro. No primeiro seminário foram discutidos os dados coletados nas entrevistas e nos sistemas de informação. Após ocorreu a construção coletiva da primeira etapa da CE - Histórico de Enfermagem (anamnese e exame físico). A etapa elabora foi testada pelos enfermeiros por 15 dias.

O segundo seminário, iniciou com discussões acerca da etapa da anamnese testada, foram decididos os ajustes e complementações necessárias. Na sequência, ocorreu a construção do roteiro para o exame físico, contemplando todos os sistemas corporais da criança de zero a dois anos de idade. Assim como no seminário anterior, o roteiro do exame físico foi testado pelos enfermeiros por 15 dias.

No terceiro seminário discutiu-se sobre o roteiro de exame físico, na sequência foi realizada a sua aprovação e em seguida a construção dos Diagnósticos (DE), Intervenções de Enfermagem (IE) e Resultados Esperados (RE), considerando a CIPE® e organizados dentro dos campos das Necessidades Humanas Básicas de Horta (2011). Porém, para essa construção foi necessário, inicialmente, realizar uma capacitação acerca da utilização desse SLP, pois apenas uma enfermeira possuía esse conhecimento.

Os DE, IE e RE foram elencados de acordo com a experiência e julgamento clínico das enfermeiras na assistência à criança e, considerando os dados extraídos dos sistemas de informação na etapa 2 (diagnóstico de situação) o que possibilitou aproximação da realidade e das necessidades locais. Ao final desse encontro ficou acordado que 0 instrumento contendo anamnese e exame físico, DE, IE e RE seria testado por 30 dias.

No quarto seminário foi discutida a versão final do instrumento de CE à criança e realizada a avaliação geral do instrumento por meio do Índice de Validade do Conteúdo (IVC).

A etapa de qualificação dos enfermeiros foi constante, proporcionada pelas discussões e construções coletivas o que instrumentalizou os enfermeiros para a implementação da CE à criança.

Publicização: para contemplar essa etapa o instrumento foi compartilhado com a Coordenação da APS e com todos os enfermeiros que atuam nos dois municípios visando sua implementação durante as $\mathrm{CE}$. Em um dos municípios, esse estudo, desencadeou a elaboração do Protocolo de Atenção à Saúde da Criança, no outro, o instrumento de CE à criança foi incorporado ao protocolo municipal vigente.

Também está publicado no E-book Produções do Mestrado Profissional em Enfermagem na Atenção Primaria à Saúde: contributos para a gestão e o cuidado (Zanatta et al., 2020).

Os dados coletados nas entrevistas e durante as discussões coletivas nos seminários, foram organizados, considerando a análise de conteúdo (Bardin, 2011) em três etapas: pré-análise; exploração e tratamento dos resultados e inferência e interpretação. 
Pré-análise: ocorreu a transcrição das entrevistas e dos seminários, organização e leitura do material empírico. Exploração e tratamento dos resultados: foram realizadas leituras em profundidade, visando identificar categorias. Inferência e interpretação dos resultados: ocorreu a aproximação dos resultados extraídos do material empírico e a discussão com a literatura científica, visando dar significado e validade aos dados brutos.

A pesquisa seguiu os preceitos éticos da Resolução no 466 de 2012 do Conselho Nacional de Saúde para pesquisa com seres humanos, aprovada pelo Comitê de Ética local sob Parecer nํ․ 2.630 .923 (02/05/2018).

\section{Resultados}

A RI resultou em 2.280 estudos, destes 1.377 foram excluídos por estarem duplicados, 417 indisponível e 447 por fuga da temática assim, 39 estudos selecionados foram lidos na íntegra e desses, 20 incluídos no corpus deste trabalho.

Os estudos foram classificados quanto ao Nível de Evidência (Melnyk \& Fineout-Overholt, 2011), ficando assim classificados: 15 estudos classificados em nível VI - proveniente de estudos descritivos ou qualitativos; três classificados em nível IV - derivados de estudos de caso-controle e de coorte; um classificado em nível II - proveniente de ensaio clínico randomizado; um estudo classificado em nível I- revisão sistemática com metanálise.

As tecnologias em saúde utilizadas no atendimento às crianças de zero a cinco anos, descritas na literatura, foram classificadas em leve, leve-duras e duras (Merhy, 2016) conforme figura 1:

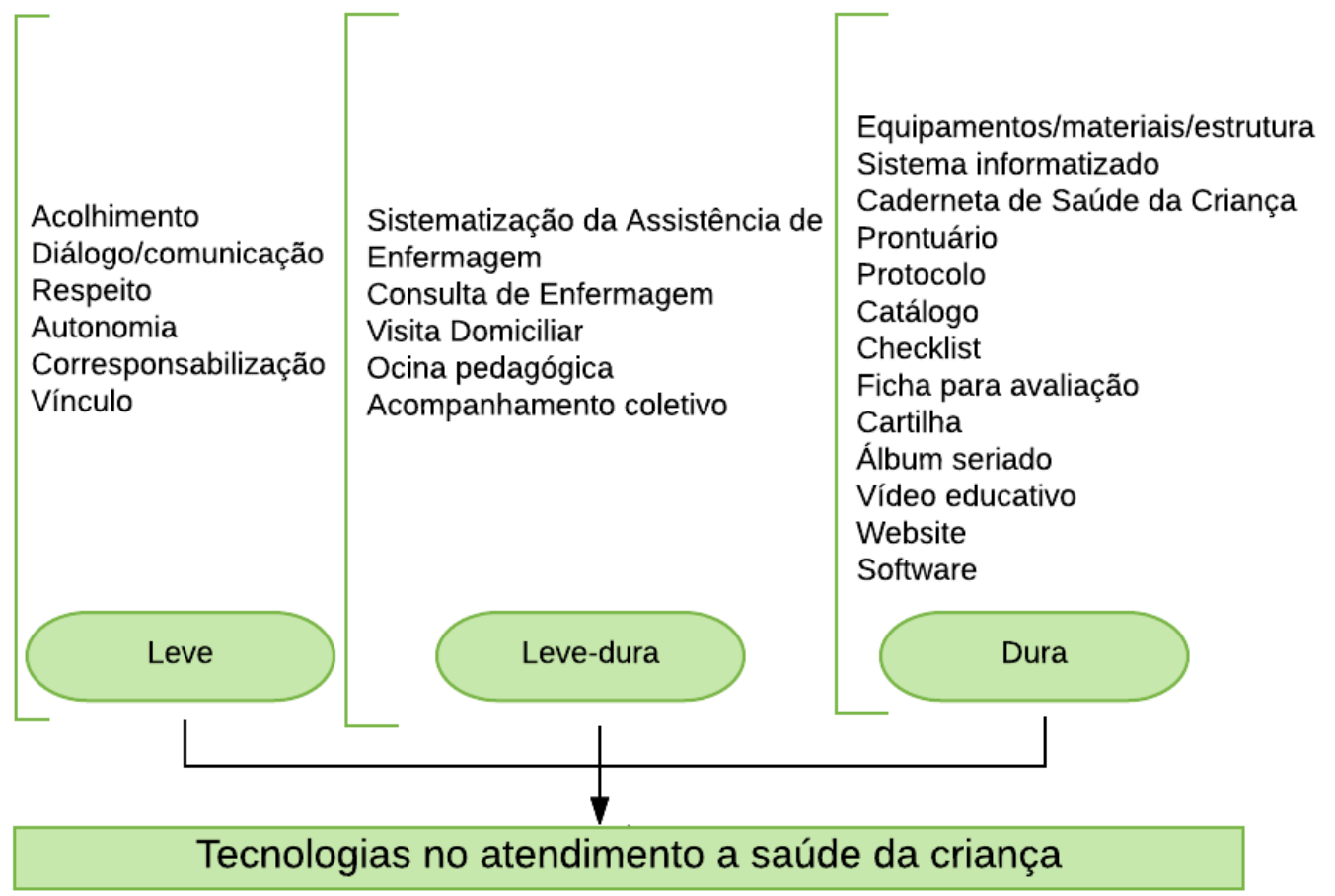

Fig. 1. Tecnologias no atendimento à criança identificadas na Revisão Integrativa da Literatura.

O perfil epidemiológico da saúde infantil, realizado nos Sistema de Informação evidenciaram como as principais causas de morbidade hospitalar em crianças menores de um ano de idade, afecções no período perinatal, doenças do aparelho respiratório, doenças infecciosas e parasitarias. Em relação aos motivos de atendimentos nas Unidades Básicas de Saúde (UBS), de ambos os municípios, foram por Nasofaringite aguda (resfriado comum), Otite externa não especificada e Doença do refluxo gastresofágico sem esofagite. 


\subsection{Consulta de Enfermagem à Criança: Compreensões e Dificuldades}

Os dados coletados nas entrevistas revelam que os enfermeiros compreendem a CE como o momento para conhecer a história pregressa e atual da criança e sua família, realizar o atendimento/avaliação. Reconhecem a necessidade de implementá-la, mas declaram dificuldades para executá-la de forma sistematizada, considerando todas as etapas.

Realizam a etapa do Histórico de Enfermagem, porém apenas a anamnese, indicam dificuldades na realização do exame físico. Apenas cinco enfermeiros, das 26 participantes, realizam as etapas de Planejamento e Avaliação. A etapa de DE é não é realizada, pois não sabem como utilizar um SLP para subsidiar sua construção.

A justificativa para a não realização de algumas etapas envolve falta de formação teórica para isso, grande rotatividade dos enfermeiros na UBS o que dificulta a implementação dessa atividade e, principalmente, a formação de vínculo com a família da criança que tem como consequência a pouca adesão das famílias para a CE. Ainda, sinalizam dificuldades quanto a estrutura física das UBS, ou seja, falta de espaço para a realização da CE. Todos relataram dificuldade de realizar a CE pela falta de um roteiro para guia-la em todas as suas etapas, bem como a falta de educação permanente para subsidiá-los para essa atividade.

\subsection{Construção e Validação do Instrumento para a CE}

Conforme acordado pelos participantes do estudo o instrumento foi construído considerando as etapas preconizadas pela Resolução 358/2009 (Cofen, 2009): Histórico de Enfermagem, Diagnósticos de Enfermagem, Planejamento e Avaliação de Enfermagem e como referencial teórico para subsidiar essa etapas foi adotada a Teoria de Wanda Horta (2011) e a CIPE®.

O Histórico de Enfermagem ficou igual nos dois locais do estudo. A anamnese considerou dados socioeconômicos e culturais, antecedentes familiares, história obstétrica pregressa e do filho em avaliação, dados do nascimento, triagens neonatais, amamentação, eliminações, sono e repouso, cuidados com higiene, interação mãe-bebê. O exame físico contemplou: pele e mucosas, cabeça e pescoço, tórax, abdômen, quadril genitália, coluna, membros superiores e inferiores e avaliação neurológica (reflexos).

As etapas de DE, IE e RE ficaram diferentes nos dois município, pois foram considerados os dados epidemiológicos de cada local e as principais queixas/motivos de atendimentos de crianças na APS. Em um município foram elencados 19 DE, 19 RE e 38 IE e no outro foram 99 DE, 99 RE e 206 IE.

Os DE, IE e RE foram construídos considerando a CIPE® e organizados dentro dos campos das Necessidades Humanas Básicas de Horta (2011) considerando necessidades psicobiológicas: oxigenação, nutrição, eliminação, sono e repouso cuidado corporal, integridade cutaneomucosa, regulação térmica regulação neurológia, regulação imunológica, regulação crescimento celular, regulação vascular e as necessidades psicossociais: segurança/amor, participação/atenção.

Após a construção os enfermeiros realizaram a avaliação do instrumento por meio de um instrumento contendo uma escala do tipo likert com pontuação de um a quatro (1Inadequado, 2- Parcialmente adequado, 3- Adequado e 4- Totalmente adequado) considerado aceitável o score igual ou superior a 0,80 de taxa de concordância (PolitO'hara \& Beck, 2011). Nesse estudo o IVC em um município foi 0,8 e no outro 1,0 . 


\section{Discussão}

Instrumentos que orientam a realização da CE são classificados como tecnologias leveduras. Estes direcionam o enfermeiro no desenvolvimento de sua prática assistencial, durante a qual fazem uso de tecnologias leves como a comunicação, a escuta e o vínculo (Merhy, 2016).

Os enfermeiros compreendem a CE como uma tecnologia e reconhecem sua importância na assistência à criança, contudo a realizam de forma fragmentada, não contemplando todas as suas etapas. Situação justificada, especialmente, pela falta de um instrumento guia e pelo pouco embasamento teórico para a execução de algumas delas. Compreendem o Histórico de Enfermagem como uma etapa importante para a construção do vínculo com a criança e sua família, oportunizando, um atendimento integral. Porém, a falta de um instrumento para a coleta das informações fragiliza a CE e, consequentemente, o atendimento de qualidade e resolutivo, sobretudo, quanto a realização do exame físico.

Segundo Crivelaro et al., (2020), a identificação dos problemas, sob o olhar da integralidade, ocorre no Histórico de enfermagem, por meio da escuta qualificada que favorece a formação do vínculo. Horta (2011) enfatiza que a coleta de dados, é justamente a primeira etapa da CE devido sua importância na coleta de informações que darão subsídios à elaboração das demais.

A etapa de DE foi relatada como a menos realizada, pela dificuldade de compreensão quanto sua construção e operacionalização, pois muitos tiveram contato apenas na graduação ou mesmo desconhecem sua elaboração. Os resultados desse estudo corroboram outras pesquisas sobre as dificuldades dos enfermeiros em construir DE (Moretti et al., 2016; Spazapan, 2017).

Para Horta (2011), a etapa de DE inicia na ação de identificar as necessidades do ser humano e avaliar qual seu grau de dependência. Observou-se que as enfermeiras partem dessa identificação, contudo apresentam dificuldade na elaboração dos DE, na utilização de um SLP e na realização de um raciocínio diagnóstico para sua utilização.

Destaca-se a relevância dos DE, elaborados a partir de um SLP como a CIPE®,para potencializar a práticas assistenciais e agregar qualidade ao cuidado (Andrada et al., 2015; Moura et al., 2015). A CE contemplada em todas as suas etapas, promove um cuidado integral à criança, a confiança, formação de vínculo com a família, estimula o retorno para consultas rotineiras e facilita a continuidade do cuidado, além de dar cientificidade ao trabalho do enfermeiro (Ribeiro et al, 2014).

A CE, realizada na sua integralidade, permite um olhar holístico à criança, um ser que possui necessidades psicobiológicas, psicossociais, psicoespirituais, intimamente interconectadas, pertencente a uma família e comunidade (Horta, 2011).

Em relação às dificuldades dos enfermeiros durante a CE à criança ressalta-se a importância e necessidade de instituir atividades de Educação Permanente em Saúde à esses profissionais, a exemplo do que foi realizado nesse estudo.

\section{Conclusões}

A pesquisa-ação oportunizou momentos de estudo, discussão, troca de experiências entre os enfermeiros e pesquisadora, fortaleceu a compreensão da importância da CE sistematizada, ancorada em referencias teóricos e metodológicos e voltada às necessidades de cada local.

O instrumento desenvolvido contribuirá para a qualificação e resolutividade da CE à criança. Poderá ser implementado e/ou adaptado para ser utilizado em diferentes os cenários da APS. 


\section{Agradecimentos}

CAPES/COFEN (financiamento via Edital № 27/2016).

\section{Referências}

Andrada, M.M.C. et al. (2015). Diagnósticos de enfermagem para idosos internados, segundo a Classificação Internacional das Práticas de Enfermagem. Rev Rene, 16 (1), 97-105. https://doi.org/ 10.15253/2175-6783.2015000100013

Bardin, L. (2016). Análise de conteúdo. Edições 70.

Conselho Federal de Enfermagem. (2009). Resolução no 358 de 2009. http://www.cofen.gov.br/resoluo-cofen-3582009 4384.htm

Crivelaro, P.M. da S. et al. (2020). Consulta de enfermagem: uma ferramenta de cuidado integral na atenção primária à saúde. Brazilian Journal of Development, 6, 49310- 4932. http://doi.org/10.34117/bjdv6n7-542.

Garcia, T.R. (Organizador). (2018). Classificação Internacional para a prática de enfermagem CIPE®: Versão 2017. Artmed.

Góes, F.G.B. et al., (2018). Contribuições do enfermeiro para boas práticas na puericultura: revisão integrativa da literatura. Revista Brasileira de Enfermagem, 71(Suppl. 6), 28082817. https://doi.org/10.1590/0034-7167-2018-0416

Horta, W.A.(2011). Processo de Enfermagem. Guanabara Koogan

Merhy, E.E. (Org.) et al. (2016). Avaliação compartilhada do cuidado em saúde: surpreendendo o instituído nas redes. Hexis.

Melnyk, B.M. \& Fineout-Overholt, E. Making the Case for Evidence-Based Practice and Cultivating a Spirit of Inquiry. Em Melnyk, B.M. \& Fineout-Overholt, E. Evidence-based practice in nursing \& healthcare: a guide to best practice. Wolters Kluwer Health, Lippincott Williams \& Wilkins.

Ministério da Saúde. (2012) Cadernos de saúde da criança: crescimento e desenvolvimento. https://bvsms.saude.gov.br/bvs/publicacoes/saude_crianca_crescimento_desenvolvimento.p df

Moreira, M.D.S., \& Gaiva, M.A.M. (2017). Approach of the child's life context of the in the nursing appointment. Rev Fund Care Online, 9, 432-440. http://dx.doi.org/10.9789/21755361.2017.v9i2.432-440

Moretti, C.A. et al. (2016). Implementação da consulta de enfermagem na Estratégia Saúde da Família: desafios e potencialidades. Journal of Nursing and Health, 6(2), 309-320. https://periodicos.ufpel.edu.br/ojs2/index.php/enfermagem/article/view/7159/6046

Moura, D. de J. M., et al. (2014). Sistematização da assistência de enfermagem fundamentada na CIPE® e na teoria da adaptação em hipertensos. Revista Eletrônica De Enfermagem, 16, 710-719. https://doi.org/10.5216/ree.v16i4.22945

Polit-O'hara, D., \& Beck, C.T. (2011). Fundamentos de pesquisa em enfermagem: avaliação de evidencias para pratica da enfermagem. Artes Medicas.

Ribeiro, D.G. et al (2014). Fatores de risco para o desenvolvimento de crianças atendidas em Unidades de Saúde da Família, ao final do primeiro ano de vida. Ciência \& Saúde Coletiva, 19, 215-226. https://doi.org/10.1590/1413-81232014191.1904

Siega, C.K, et al., (2020). Lived Experiences and meanings of the Nurse Consultation in Childcare: analysis in the light of Wanda Horta. Rev. Enferm. UFSM, 10, e65, 1-20. https://doi.org/10.5902/2179769241597 
Spazapan, M.P. (2017). Processo de enfermagem na atenção primária: percepção de enfermeiros de Campinas-SP. [Dissertação Mestrado, Universidade Estadual de Campinas].

https://sucupira.capes.gov.br/sucupira/public/consultas/coleta/trabalhoConclusao/viewTrabal hoConclusao.jsf?popup=true\&id_trabalho $=5219937$

Thiollent, M. (2011). Metodologia da pesquisa-ação. Cortez.

Yakuwa, M.S et al. (2018) Nursing strategies for child health surveillance. Rev. Latino-Am. Enfermagem, 26, 1-8. http://dx.doi.org/10.1590/ 1518-8345.3007.2434.

Zanatta, E.A. et al. (2020). Produção do mestrado profissional em enfermagem na atenção primária à saúde: contributos para a gestão e o cuidado. http://sistemabu.udesc.br/pergamumweb/vinculos/000081/00008166.pdf

Zocche et al., (2018). Protocolo para revisão integrativa: caminho para busca de evidências. Em Teixeira E (organizadora). Desenvolvimento de tecnologias cuidativo-educacionais. (pp. 237249).Moriá. 